\title{
Targeting of EZH2 inhibits epithelial-mesenchymal transition in head and neck squamous cell carcinoma via regulating the STAT3/VEGFR2 axis
}

\author{
MINGHUI ZHAO $^{1 *}$, XIAOMENG HU $^{1 *}$, YINI XU $^{1 *}$, CHUANQIANG WU $^{1}$, JINLIANG CHEN $^{1}$, \\ YU REN ${ }^{2}$, LINGPING KONG $^{1}$, SHANSHAN SUN ${ }^{1}$, LUN ZHANG $^{1}$, RUI JIN ${ }^{1}$ and XUAN ZHOU ${ }^{1}$
}

\begin{abstract}
${ }^{1}$ Department of Maxillofacial and Otorhinolaryngology Oncology, Tianjin Medical University Cancer Institute and Hospital, Key Laboratory of Cancer Prevention and Therapy, Tianjin Cancer Institute, National Clinical Research Center of Cancer, Tianjin 300060; ${ }^{2}$ Research Center of Basic Medical Science, Tianjin Medical University, Tianjin 300070, P.R. China
\end{abstract}

Received February 23, 2019; Accepted August 19, 2019

DOI: 10.3892/ijo.2019.4880

\begin{abstract}
Tumor metastasis regulated by epithelial-mesenchymal transition (EMT) plays a significant role in the development of human cancers, whereas the molecular mechanisms of this process in head and neck squamous cell carcinoma (HNSCC) remain elusive. In this study, we found that inhibition of enhancer of zeste homolog 2 (EZH2) resulted in suppressed EMT in HNSCC in vitro and in vivo. We reported that signal transducer and activator of transcription factor 3 (STAT3)/vascular endothelial growth factor receptor 2 (VEGFR2) axis served as the downstream signaling of EZH2 and mediated EMT in HNSCC. EZH2 inhibition downregulated the expression of key molecules of the STAT3/VEGFR2 axis and EMT-related markers, while the expression of E-cadherin was upregulated in HNSCC cells. Targeting the EZH2/STAT3/VEGFR2 axis significantly reduced motility of HNSCC cells. Furthermore, EZH2 knockdown reduced the growth of xenograft HNSCC tumors via inhibiting the
\end{abstract}

Correspondence to: Dr Xuan Zhou or Dr Rui Jin, Department of Maxillofacial and Otorhinolaryngology Oncology, Tianjin Medical University Cancer Institute and Hospital, Key Laboratory of Cancer Prevention and Therapy, Tianjin Cancer Institute, National Clinical Research Center of Cancer, Tianjin 300060, P.R. China

E-mail: byron2000zhou@sina.com

E-mail: ruijin123@sina.com

*Contributed equally

Abbreviations: DZNep, 3-deazaneplanocin A; EMT, epithelialmesenchymal transition; EZH2, enhancer of zeste homolog 2; H3K27me3, trimethylation of lysine 27 on histone H3; HNSCC, head and neck squamous cell carcinoma; STAT3, signal transducer and activator of transcription factor 3; VEGF, vascular endothelial growth factor; VEGFR2, vascular endothelial growth factor receptor 2; KDR, kinase insert domain receptor

Key words: EZH2, HNSCC, STAT3, VEGFR2, EMT, metastasis
EZH2/STAT3/VEGFR2 axis. In conclusion, we proposed that EZH2 regulates EMT and tumor invasion and metastasis in HNSCC by governing the STAT3/VEGFR2 axis. These findings provide a rationale for developing novel strategies to treat invasive and metastatic HNSCC via targeting the EZH2/STAT3/VEGFR2 pathway.

\section{Introduction}

Approximately 600,000 new cases of head and neck squamous cell carcinoma (HNSCC) are diagnosed worldwide annually, making this cancer the sixth most common human malignancy (1). Systemic therapies have been employed for treating this cancer with significant progress; however, the 5-year overall survival remains low at $\sim 50 \%$ (2), due to subsequent metastasis developed in such patients which accounts for the poor clinical outcome of HNSCC (2). Epithelial mesenchymal transition (EMT), initially described in embryonic development, has been reported to be one of the most important mechanisms of cell migration and invasion in physiological and pathological processes, such as tissue repair, fibrosis and cancer progression (3). When cells undergo EMT, they may change their morphology and lose cell-cell junctions, while retaining expression of molecules that promote migration and invasion (3). Of note, EMT leads to the poor prognosis of HNSCC $(4,5)$.

Enhancer of zeste homolog 2 (EZH2), a catalytic subunit of the polycomb repressive complex 2 , serves an important role in the modification of chromatin structure primarily by trimethylating the lysine 27 residue of histone $\mathrm{H} 3$ (H3K27me3) $(6,7)$. EZH2 is upregulated in several cancer types, including HNSCC and serves as a negative prognostic factor (8-11). Reports showed that EZH2 could regulate tumor angiogenesis (12), apoptosis (11), EMT (13), and cell migration and invasion $(14,15)$. Our previous research revealed that inhibition of EZH2 with 3-deazaneplanocin A (DZNep), a novel inhibitor of EZH2, induced the apoptosis of HNSCC cells via mitochondria-dependent cell death (11). EZH2 is therefore a promising therapeutic target for treating HNSCC. 
Signal transducer and activator of transcription factors 3 (STAT3) is aberrantly activated in $70 \%$ of all cancer types $(16,17)$, and is involved in cancer cell proliferation, angiogenesis, invasion, and the survival and maintenance of cancer stem cells (18). STAT3 is constitutively activated during tumor progression and metastasis, and the level of tyrosine-phosphorylated STAT3 (Try705) is associated with the poor prognosis of HNSCC (19). Thus, STAT3 is a potential therapeutic target for treating HNSCC. A previous study reported that phosphorylation of EZH2 at serine 21 may enhance STAT3 activity by direct binding to and methylating STAT3 (20). Furthermore, EZH2 could mediate the oncogenic activity of STAT3 through demethylation of K49 (21); however, whether and how EZH2 interacts with STAT3 in HNSCC remains unclear.

Vascular endothelial growth factor receptor 2 [VEGFR2, kinase insert domain receptor (KDR)] is the major receptor for vascular endothelial growth factor (VEGF) and is upregulated in HNSCC (22). Emerging evidence has suggested that STAT3 may participate in the VEGF/VEGFR2 signaling cascade in human cancers (23). For example, STAT3 activation has been reported to promote angiogenesis through upregulation of VEGF expression in lung cancer (24). Notably, a previous study demonstrated that VEGF upregulated is induced by STAT3 activation and thus promotes brain metastasis in human melanoma (25). Based on these studies, we investigated whether EZH2 mediates metastasis and the EMT process in HNSCC via regulating the STAT3/VEGFR2 axis. We aimed to characterize the EZH2-mediated EMT pathway to provide a rationale for developing novel therapeutic strategies to suppress HNSCC metastasis.

\section{Materials and methods}

Cell culture and reagents. The human HNSCC cell line CAL27 was purchased from the American Type Culture Collection and the UM1 cell line was a gift from Professor Jinsong Hou of Guanghua School of Stomatology, Hospital of Stomatology, Sun Yat-sen University. Cells were maintained in Dulbecco's Modified Eagle's medium (DMEM)/Ham's F-12 and DMEM supplemented with $10 \%$ fetal bovine serum (FBS; Gibco; Thermo Fisher Scientific, Inc.) at $37^{\circ} \mathrm{C}$ in a $5 \% \mathrm{CO}_{2}$ humidified incubator. UM1 and CAL27 cells were treated with DZNep $\left(2,5\right.$ or $10 \mu \mathrm{mol} / 1$, Selleck Chemicals) for $48 \mathrm{~h}$ at $37^{\circ} \mathrm{C}$ to inhibit EZH2 expression. DZNep was dissolved in dimethyl sulfoxide (DMSO; Sigma-Aldrich, Merck KGaA). UM1 and CAL27 cells were treated with DMSO as control.

Small interfering RNA (siRNA) and plasmid transfection.UM1 and CAL27 cell lines were transfected with siRNA-negative control (siNC) or siRNAs (20 nmol/1, Guangzhou Ribobio Co., Ltd.) against STAT3 or VEGFR2, labeled as siNC (5'-UUC UCCGAACGUGUCACGU-3'), siSTAT3 (5'-AGTCAGGTT GCTGGTCAAA-3') or SiKDR (5'-GGTAAAGATTGATGA AGAA-3'), respectively, using Lipofectamine ${ }^{\circledR} 2000$ reagent according to the manufacturer's instructions (Invitrogen; Thermo Fisher Scientific, Inc.). For the transient overexpression of EZH2, the pLVX-IRES lentiviral vector system was used to deliver EZH2 gene into UM1 and CAL27 cells, the empty pLVX plasmid (Shanghai GeneChem Co., Ltd.) was used as a control. FBS-free Opti-MEM medium (Gibco; Thermo Fisher Scientific, Inc.) was employed for transfection, after $6 \mathrm{~h}$, the medium was replaced by DMEM/Ham's F-12 or DMEM. Subsequent analysis were carried out after transfection for $48 \mathrm{~h}$.

Western blotting. Cell lysates were prepared in radioimmunoprecipitation assay buffer with protease and phosphatase inhibitors (Beijing Solarbio Science \& Technology Co., Ltd.) $48 \mathrm{~h}$ after the treatments of cells. Protein concentrations were determined by using a BCA protein assay kit (Micro BCA Protein Assay kit; Thermo Fisher Scientific, Inc.). A total of $30 \mu \mathrm{g}$ of each protein samples was separated in an analytical 10 or $15 \%$ SDS-PAGE and then transferred onto polyvinylidene difluoride membranes (Merck KGaA). The membranes were blocked in blocking buffer (5\% non-fat milk, $0.1 \%$ Tween 20 in PBS) for $2 \mathrm{~h}$ at room temperature and then incubated overnight at $4^{\circ} \mathrm{C}$ with primary antibodies $(1: 1,000)$ against human EZH2, STAT3, phosphorylated (p)-STAT3 (Tyr705), E-cadherin, $\mathrm{N}$-cadherin, Vimentin, Twist-related protein 1 (Twist-1) obtained from Cell Signaling Technology, Inc.; primary antibodies against VEGF, VEGFR2 (Abcam), and GAPDH (OriGene Technologies, Inc.) were employed (Table I). Mouse (sc-2005) or rabbit (sc-2004) IgG antibodies coupled with horseradish peroxidase (Santa Cruz Biotechnology, Inc.) were used as secondary antibodies $(1: 5,000)$, which were applied at room temperature for $2 \mathrm{~h}$. Chemiluminescent horseradish peroxidase substrate (Merck $\mathrm{KGaA}$ ) was used as the visualization reagent following the instructions. The densitometry of the immunoblots was determined using ImageJ software (version 5.2.5; National Institutes of Health).

Wound-healing and Transwell assay. Approximately $2 \times 10^{5}$ UM1 cells or $5 \times 10^{5}$ CAL27 cells per well were added into 6-well plate. A linear scratch was made by $10 \mu \mathrm{l}$ pipette tips. The plates were incubated in serum-free medium. Images were taken at 0 and $24 \mathrm{~h}$ from several randomly selected wound locations. Cell invasion and migration assays were performed using Transwell membranes coated with Matrigel (BD Biosciences) or left uncoated, respectively. The lower chamber was filled with medium containing with $20 \%$ FBS (Gibco; Thermo Fisher Scientific, Inc.) and cultured at $37^{\circ} \mathrm{C}$ for $24 \mathrm{~h}$. The non-invading cells were removed with cotton swabs and invading cells were fixed with $4 \%$ paraformaldehyde for $15 \mathrm{~min}$ at room temperature and then stained with $0.1 \%$ crystal violet (both from Beijing Solarbio Science \& Technology Co., Ltd.) for $30 \mathrm{~min}$ at room temperature. Each image was observed using an inverted microscope (DMI6000B; Leica Microsystems, Inc.) at x40 magnification, and cells were counted in five separate fields in three independent experiments.

Immunofluorescence staining. For immunofluorescence staining, $5 \times 10^{4}$ cells/well UM1 or CAL27 were seeded on the coverslips and fixed with $4 \%$ paraformaldehyde (Beijing Solarbio Science \& Technology Co., Ltd.) for $15 \mathrm{~min}$ at room temperature. Then, the cells were permeabilized with $0.2 \%$ Triton-X 100 for $10 \mathrm{~min}$ and treated with $1 \%$ bovine serum albumin (Beijing Solarbio Science \& Technology Co., Ltd.) for $1 \mathrm{~h}$. Immunofluorescence staining was conducted with 
Table I. Primary antibodies used in the present study.

\begin{tabular}{lclc}
\hline Primary antibody & Cat. no. & Supplier & Application \\
\hline EZH2 & 5246 & Cell Signaling Technology, Inc. & WB/IHC \\
STAT3 & 30835 & Cell Signaling Technology, Inc. & WB/IHC \\
p-STAT3 (Tyr705) & 9145 & Cell Signaling Technology, Inc. & WB/IHC \\
E-cadherin & 14472 & Cell Signaling Technology, Inc. & WB/IF/IHC \\
N-cadherin & 13116 & Cell Signaling Technology, Inc. & WB/IF/IHC \\
Vimentin & 5741 & Cell Signaling Technology, Inc. & WB/IF/IHC \\
Twist-1 & 46702 & Cell Signaling Technology, Inc. & WB/IHC \\
VEGF & ab155944 & Abcam & WB/IHC \\
VEGFR2 & ab11939 & Abcam & WB \\
GAPDH & TA-08 & OriGene Technologies, Inc. &
\end{tabular}

IHC, immunohistochemistry; STAT3, signal transducer and activator of transcription 3; p-STAT3, phosphorylated-STAT3; VEGF, vascular endothelial growth factor; VEGFR2, vascular endothelial growth factor receptor 2; WB, western blotting.

antibodies (Table I) against E-cadherin, N-cadherin, Vimentin (1:100; Cell Signaling Technology, Inc.) and F-actin was detected by phalloidin (cat no. 40734ES75; 1:200; Yeasen Biotech Co., Ltd.) overnight at $4^{\circ} \mathrm{C}$. The cells were washed with PBS and incubated with Alexa Fluor ${ }^{\circledR} 488$ (anti-rabbit, cat. no. 4412 or anti-mouse: cat. no. 4408) or Alexa Fluor 594 (anti-rabbit, cat. no. 8889 or anti-mouse, cat. no. 8890) secondary antibodies (1:500 dilutions; Cell Signaling Technology, Inc.) for $1 \mathrm{~h}$ at room temperature. Nuclei were stained using DAPI reagent (Thermo Fisher Scientific, Inc.) for $10 \mathrm{~min}$ at room temperature. Cells were visualized using FV-1000 laser scanning confocal microscope (Olympus Corporation) in five separate fields; a x100 objective lens was used in this experiment.

CAL27 xenograft tumor model and in vivo treatment. All animal protocols were approved by the Tianjin Medical University Animal Care and Use Committee and also conformed to the guidelines published by the National Institutes of Health for the care and use of laboratory animals (26). A total of $2 \times 10^{6}$ CAL27 cells were implanted subcutaneously on the right groin region of 4-week-old male nude BALB/c mice ( $\mathrm{n}=25$; weight, $\sim 15 \mathrm{~g}$ ). The housing conditions for mice were as following: Temperature, 18-22 ${ }^{\circ} \mathrm{C}$; humidity, 50-60\%; ventilation, 15 times $/ \mathrm{h}$; $12 / 12 \mathrm{~h}$ light/dark cycle; free access to food/water. After 30 days following tumor establishment, the mice were randomly divided into five groups ( $\mathrm{n}=5$ per group). Mice in individual groups received an intra-tumoral injection of designated treatment every three days with $0.07 \mathrm{mg} / \mathrm{kg}$ DZNep (DZNep group), $10 \mathrm{mg} / \mathrm{kg}$ Stattic (Stattic group), $0.07 \mathrm{mg} / \mathrm{kg}$ DZNep + $10 \mathrm{mg} / \mathrm{kg}$ Stattic (DZNep + Stattic group), $10 \mathrm{mg} / \mathrm{kg}$ Apatinib (Apatinib group; Stattic and Apatinib were purchased from Selleck Chemicals), or DMSO (DMSO group). Tumor volume (volume=long diameter $\mathrm{x}$ short diameter ${ }^{2} / 2$ ) and mice weights were determined by calipers and electronic weighing meter. After 3 weeks of observation, the mice were sacrificed and the xenograft tumors were removed for further pathological examination.

Immunohistochemistry (IHC), and hematoxylin and eosin (H\&E) staining. For IHC staining, xenograft tumor samples were fixed with $4 \%$ paraformaldehyde for $24 \mathrm{~h}$ at room temperature. Paraffin-embedded samples were sliced into $4 \mu \mathrm{m}$ thickness, and then deparaffinized, rehydrated, and incubated with primary antibodies (see Table I) against EZH2, STAT3, p-STAT3 (Tyr705), E-cadherin, N-cadherin, Vimentin, VEGF or VEGFR2 (1:100) overnight at $4^{\circ} \mathrm{C}$. Then, the tumor slices were incubated with a biotin-labeled secondary antibody (ready to use; PV-9000; OriGene Technologies, Inc.) for $1 \mathrm{~h}$ at $37^{\circ} \mathrm{C}$. The IHC (11) and H\&E (27) protocols were performed as previously described. Images were captured under an inverted microscope (DMI6000B; Leica Microsystems, Inc.) at x200 magnification in three separate fields.

Statistical analysis. SPSS software 18.0 (SPSS, Inc.) was used to analyze all data. The results were presented as the mean \pm standard deviation. Statistical evaluation between two groups was performed using a Student's t-test. Differences among multiple groups were determined by two-way ANOVA followed by a Dunnett's test. $\mathrm{P}<0.05$ was considered to indicate a statistically significant difference.

\section{Results}

Inhibition of the EZH2/STAT3 axis attenuates VEGFR2 expression in HNSCC cell lines in vitro. Our previous research showed that HNSCC cell lines UM1 and CAL27 possess high expression of EZH2, STAT3 and p-STAT3 (Tyr705) $(11,28)$, we therefore selected UM1 and CAL27 cells for further investigation. We used DZNep, a specific EZH2 small-molecule inhibitor, to suppress EZH2 expression in UM1 and CAL27 cells. The optimal dose of DZNep treatment in HNSCC cells was demonstrated in our previous report (11). In DZNeptreated UM1 and CAL27 cells, the expression levels of EZH2, p-STAT3 (Tyr705), VEGF, and VEGFR2 were markedly decreased than in untreated control cells in a dose-dependent manner; however, the expression of total STAT3 was notably unaffected (Fig. 1A).

To further investigate the importance of increased EZH2 expression in HNSCC cells, we transfected the pLVX-EZH2 expression plasmid into UM1 and CAL27 cells. As shown in 


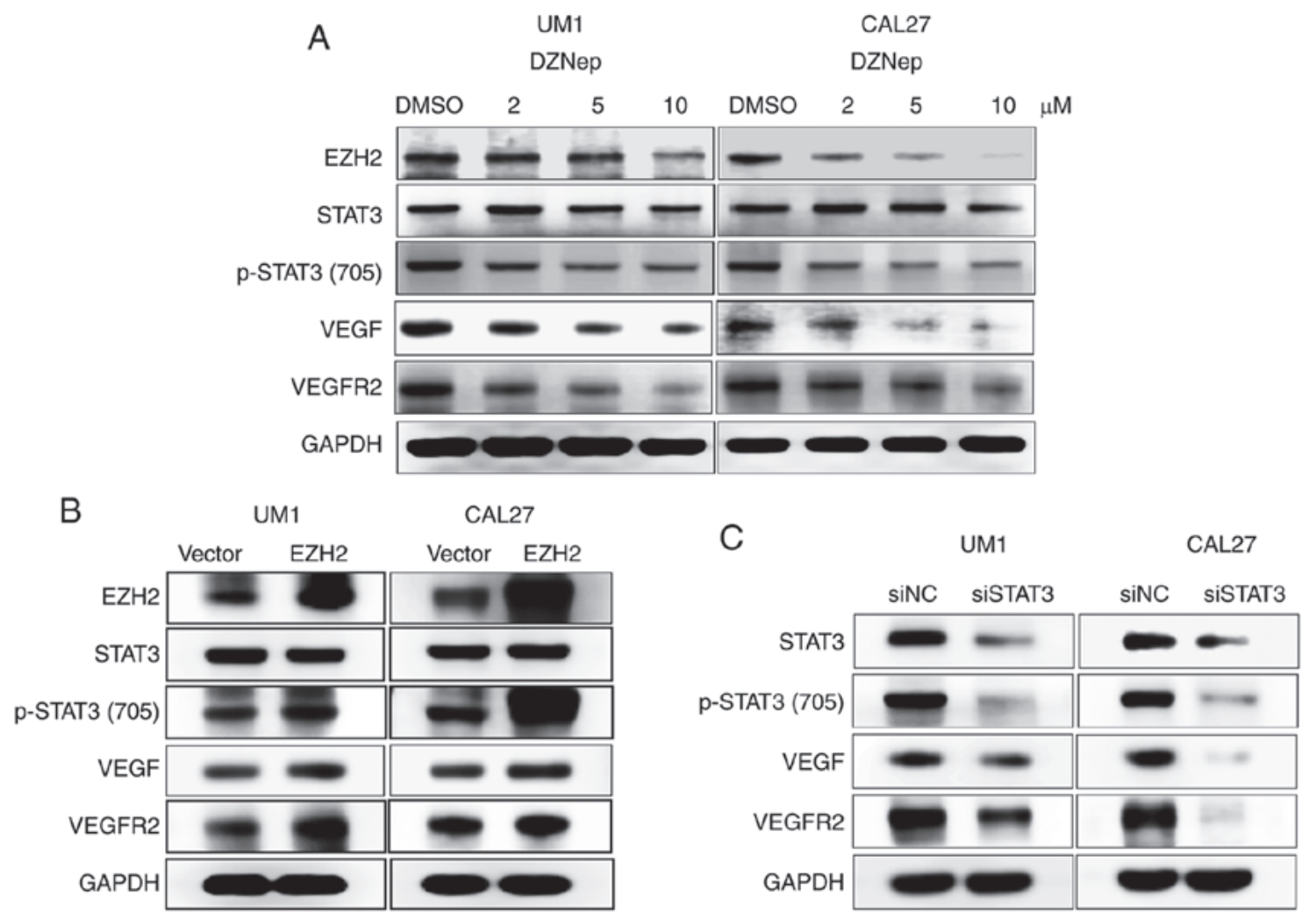

Figure 1. EZH2/STAT3 axis regulates VEGFR2 expression in HNSCC cells. (A) UM1 and CAL27 cell lines were treated with DZNep at designated concentrations $(2,5$ or $10 \mu \mathrm{mol} / \mathrm{l})$ for $48 \mathrm{~h}$. Western blot analyses were performed to investigate the expression of EZH2, STAT3, p-STAT3 (Tyr705), VEGF, and VEGFR2. GAPDH was used as a loading control. (B) HNSCC cells were transfected with pLVX-EZH2 expression plasmids, western blot analyses were performed to investigate the effects of EZH2 on the expression of p-STAT3 (Tyr705), VEGF, and VEGFR2. (C) siSTAT3 transfection was performed to determine the impact of STAT3 on p-STAT3 (Tyr705), VEGF, and VEGFR2 expression in UM1 and CAL27 cells. EZH2, enhancer of zeste homolog 2; HNSCC, head and neck squamous cell carcinoma; NC, negative control; p, phosphorylated; si, small interfering RNA; STAT3, signal transducer and activator of transcription factor 3; VEGF, vascular endothelial growth factor; VEGFR2, VEGF receptor 2.

Fig. 1B, induced EZH2 expression resulted in the increased expression of p-STAT3 (Tyr705), VEGF, and VEGFR2, with no notable differences in total STAT3 expression. Additionally, we used STAT3 siRNA to block STAT3 and p-STAT3 (Tyr705) expression. After transfection for $48 \mathrm{~h}$, the expression levels of STAT3 and p-STAT3 (Tyr705) were markedly decreased in both cell lines. Similarly, lower expression of VEGF and VEGFR2 was observed after STAT3 knockdown (Fig. 1C). These results suggest that the EZH2/STAT3 axis could regulate VEGFR2 expression in HNSCC cells.

Inhibition of EZH2 attenuates the migration, invasion, and EMT of HNSCC cells in vitro. We next used DZNep to antagonize EZH2 in UM1 and CAL27 cells. Treatment with DZNep for $24 \mathrm{~h}$ resulted in significantly delayed wound healing in both cell lines as determined via scratch tests, compared with the control $(\mathrm{P}<0.001 ;$ Fig. $2 \mathrm{~A})$. The Transwell assay showed that the number of migrating and invading cells in the DZNep-treated group was significantly reduced compared with the control ( $\mathrm{P}<0.001$; Fig. 2B). These results suggest that knockdown of EZH2 inhibits the invasion and migration of HNSCC cells. EMT involves a complex change in the cell phenotype, and is regarded as one of the most important mechanisms for cell migration and cancer metastasis $(3,29)$. Cells undergoing EMT often display cadherin switching, for example, loss of epithelial cell adhesion and tight regulation of cadherin adhesion (3).
We hypothesized that EZH2 inhibition could reduce the invasion and migration of HNSCC cells via EMT interruption. Western blotting revealed that in DZNep-treated group the EMT marker, E-cadherin, was markedly upregulated, while $\mathrm{N}$-cadherin, Vimentin and Twist-1 were notably downregulated in a dose-dependent manner compared with the control (Fig. 2E). Furthermore, immunofluorescence staining showed similar findings to the western blot assay; in DZNep-treated group, E-cadherin expression had increased, while N-cadherin and Vimentin were decreased (Fig. 2C). In the DZNep-treated group, disrupted F-actin stress fiber networks were observed with notable phenotypic changes in UM1 and CAL27 cells (Fig. 2D). Taken together, these results demonstrated that EZH2 inhibition attenuates the aggressive behavior of HNSCC cells by interrupting the EMT process.

Inhibition of STAT3 attenuates HNSCC cell migration, invasion and EMT in vitro. Since EZH2 mediates the EMT process of HNSCC cells as suggested by our results (Fig. 2), we aimed to determine whether downstream effector molecules of EZH2 participate in EMT. As shown in Fig. 1A and B, STAT3 acted as a downstream target of EZH2, we investigated whether EZH2 mediates EMT in HNSCC cells through regulating STAT3. To inhibit STAT3 activity, we transfected UM1 and CAL27 cells with siSTAT3. After $48 \mathrm{~h}$ of transfection, the expression of STAT3 and p-STAT3 (Tyr705) were significantly decreased 
A
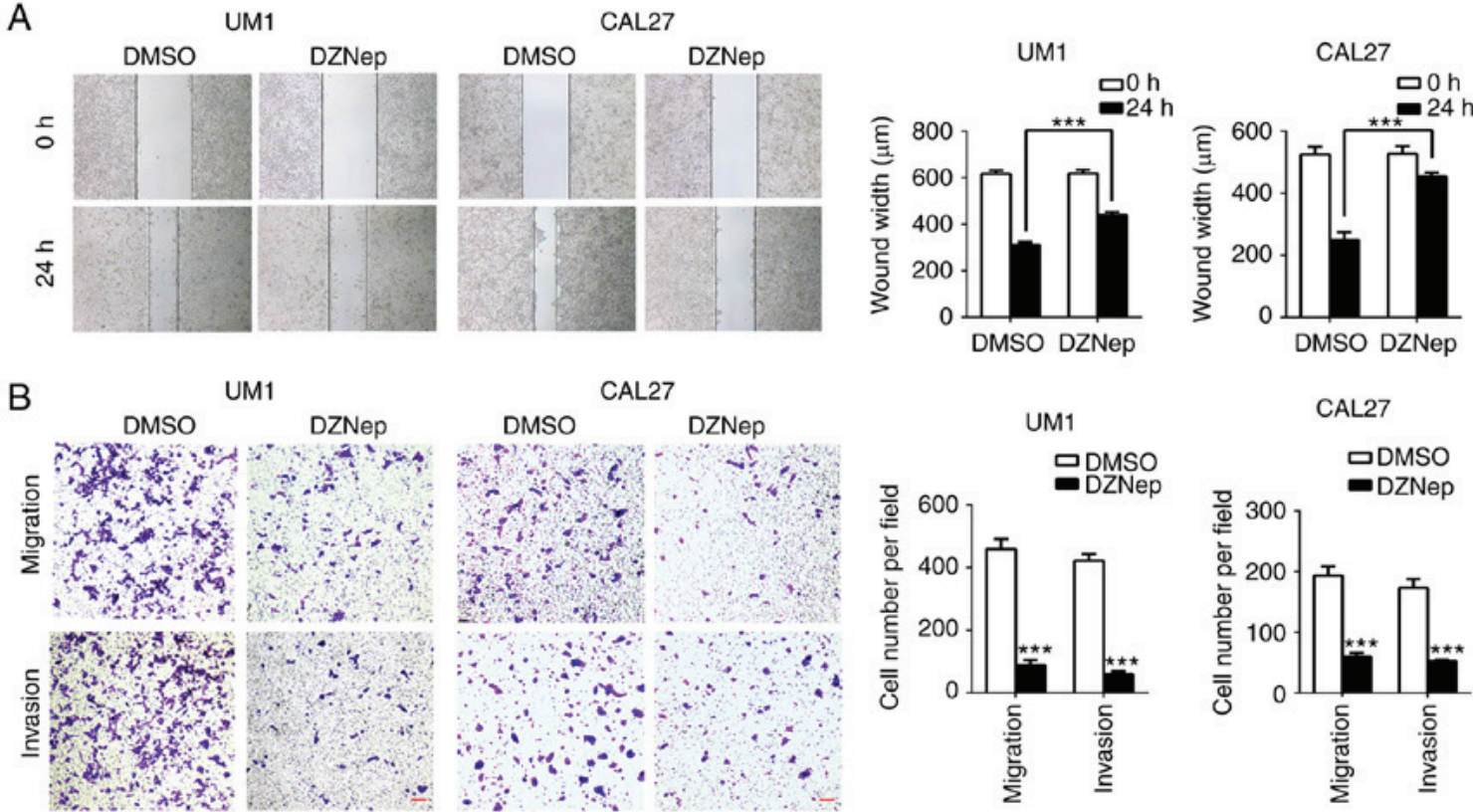

C

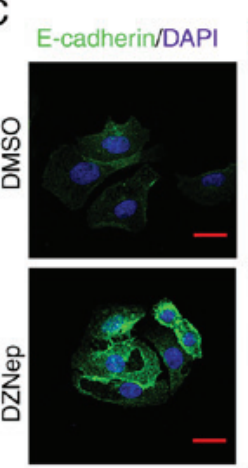

UM1
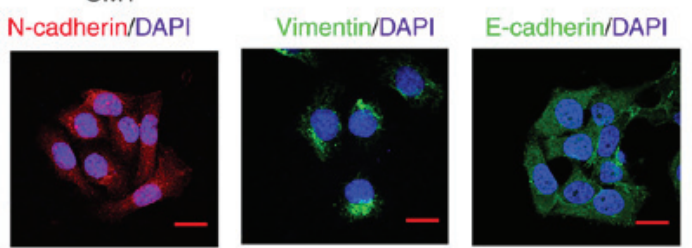

CAL27
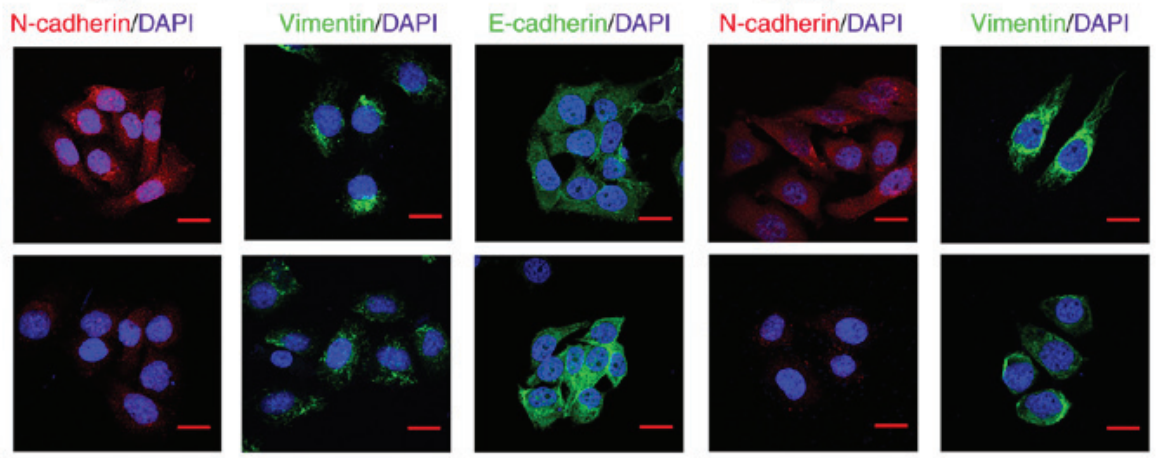

D

UM1

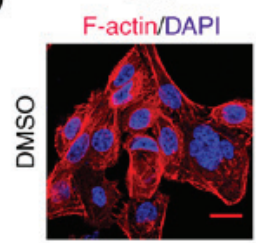

F-actin/DAP

E

UM1

DZNep

CAL27
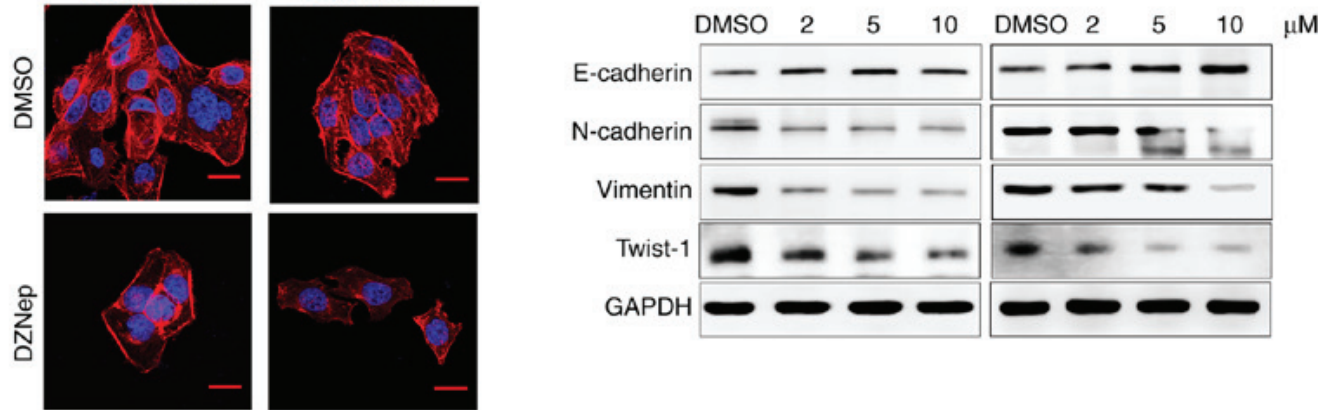

GAPD

-

Figure 2. Inhibition of enhancer of zeste homolog 2 suppresses EMT of head and neck squamous cell carcinoma cells in vitro. (A) Wound-healing assays were performed to analyze the migration in DZNep-treated UM1 and CAL27 cells. Student's t-test ${ }^{* * *} \mathrm{P}<0.001$. (B) Transwell assays were performed to investigate migration (without Matrigel) and invasion (with Matrigel) in the DZNep-treated group of both cell lines. Student's t-test. ${ }^{* * *} \mathrm{P}<0.001 \mathrm{vs}$. DMSO. Scale bar, $50 \mu \mathrm{m}$. (C) EMT-related proteins were nalyzed by immunofluorescence staining in UM1 and CAL27 cells after DZNep or DMSO treatment. Scale bar, $20 \mu \mathrm{m}$. (D) F-actin was detected by staining with phalloidin. In DZNep-treated group, F-actin was remodeled and polarized, whereas in the DMSO group F-actin presented a stress pattern. Scale bar, $20 \mu \mathrm{m}$. (E) Western blot assays were performed to show the expression of EMT-related proteins after treatment with DZNep (2, 5 or $10 \mu \mathrm{mol} /)$. DMSO, dimethyl sulfoxide; DZNep, 3-deazaneplanocin A; EMT, epithelial-mesenchymal transition; NC, negative control; si, small interfering RNA; STAT3, signal transducer and activator of transcription factor 3; STAT3, signal transducer and activator of transcription factor 3; Twist-1, Twist-related protein 1.

in both UM1 and CAL27 cell lines compared with the control (Fig. 1C). Notably, STAT3 knockdown significantly inhibited HNSCC cells migration compared with the scramble transfected cells as suggested by a scratch-wound assay $(\mathrm{P}<0.001$; Fig. 3A). Additionally, a Transwell chamber assay confirmed that siSTAT3 significantly inhibited the invasion and migration of HNSCC cells in vitro compared with the control $(\mathrm{P}<0.001$; Fig. 3B). We next determined whether STAT3 participates in the EMT process of UM1 and CAL27 cells by western blotting. After STAT3 knockdown, the expression of the EMT 
A
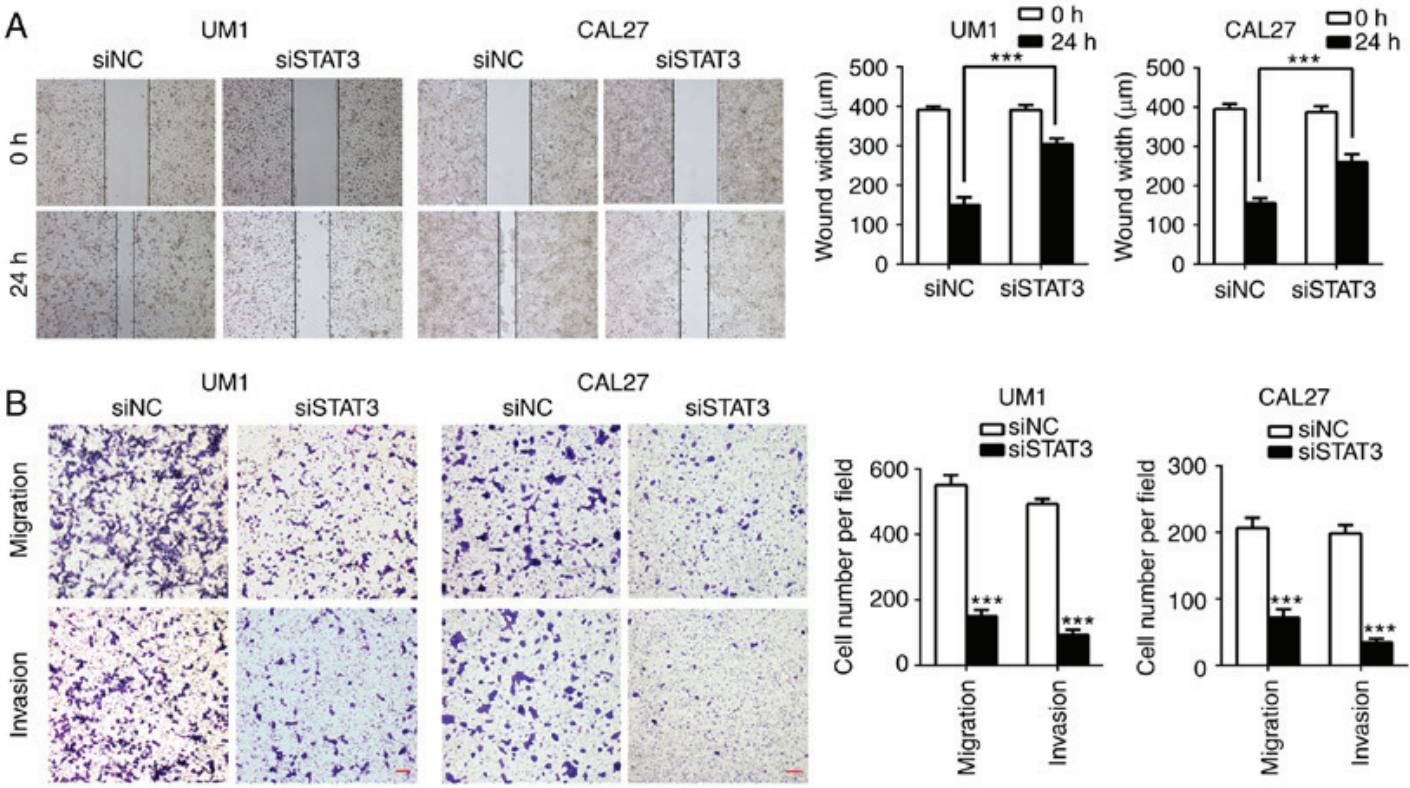

C

UM1
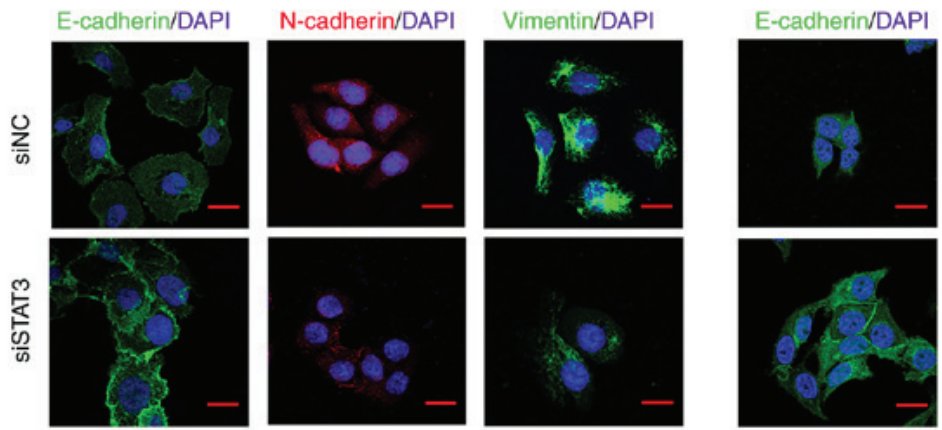

CAL27
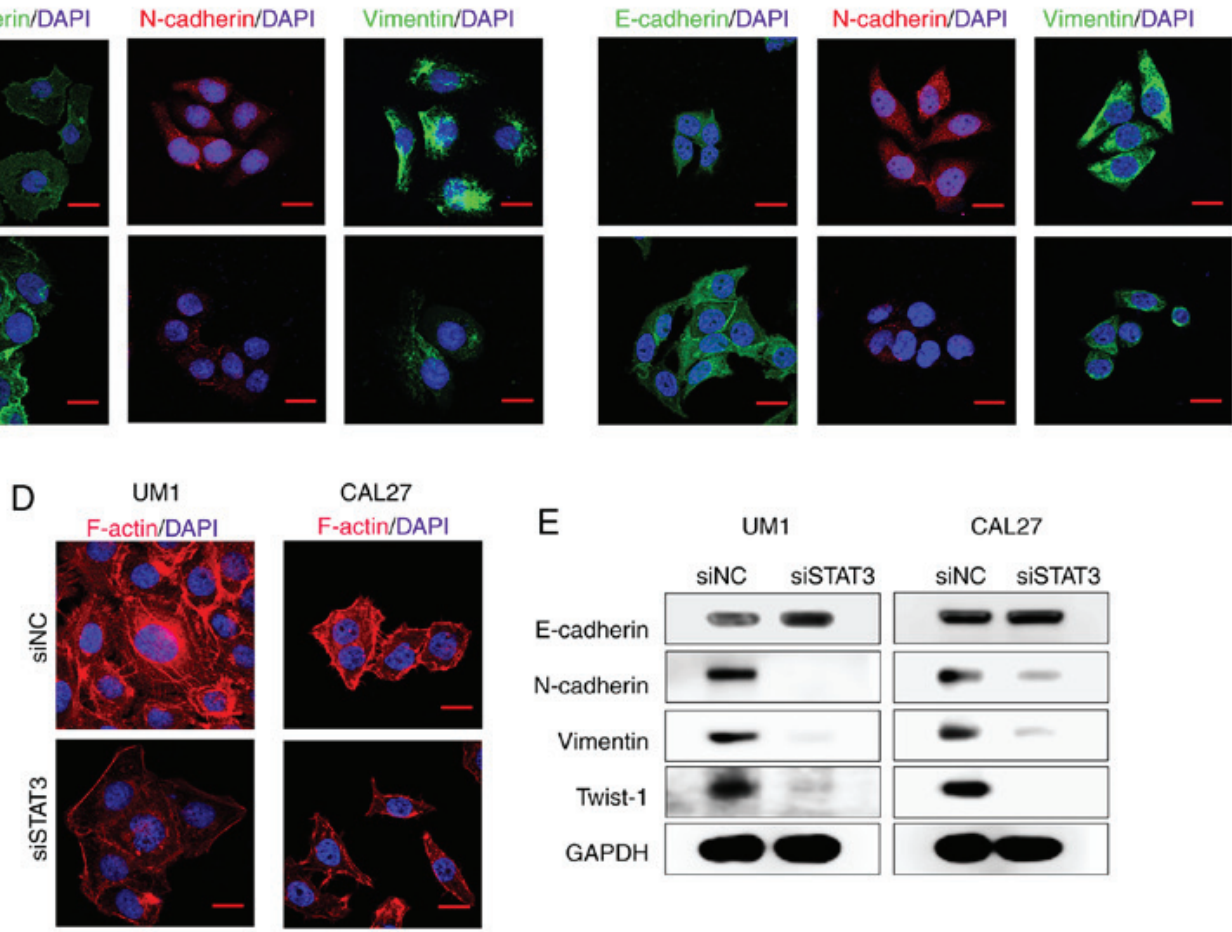

E

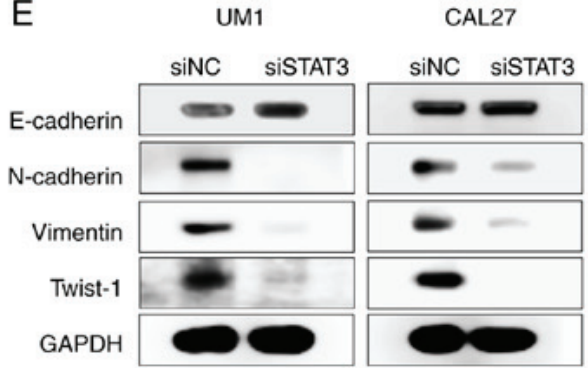

Figure 3. STAT3 inhibition suppresses the EMT of HNSCC cells in vitro. (A) Wound-healing assays performed with siSTAT3-treated UM1 and CAL27 cells, compared with scramble HNSCC cells. ${ }^{* * *} \mathrm{P}<0.001$. (B) Transwell assays performed to show migration (without Matrigel) and invasion (with Matrigel) in siSTAT3-treated group. ${ }^{* * *} \mathrm{P}<0.001$ vs. siNC. Scale bar, $50 \mu \mathrm{m}$. (C) Confocal immunofluorescence analysis performed to show the change of EMT-related proteins in siSTAT3-treated cells compared with the scramble cells. Scale bar, $20 \mu \mathrm{m}$. (D) Confocal immunofluorescence analysis performed to show F-actin rearrangement in STAT3 siRNA-treated HNSCC cells. Scale bar, $20 \mu \mathrm{m}$. (E) Western blot analysis was performed to show the effects of STAT3 knockdown on the expression of E-cadherin, N-cadherin, Vimentin and Twist-1 in UM1 and CAL27 cells. EMT, epithelial-mesenchymal transition; HNSCC, head and neck squamous cell carcinoma; siSTAT3, STAT3, small interfering RNA signal transducer and activator of transcription factor 3; Twist-1, Twist-related protein 1.

marker, E-cadherin, was markedly increased, while that of $\mathrm{N}$-cadherin, Vimentin and Twist-1 was decreased, compared with the control (Fig. 3E). Immunofluorescence staining for the EMT-related markers E-cadherin, $\mathrm{N}$-cadherin and Vimentin, further validated the results of western blot assays (Fig. 3C). Furthermore, F-actin presented a notable stress fiber pattern in HNSCC cells treated with siSTAT3 compared with the control cells (Fig. 3D). Overall, our results indicated that knockdown of
STAT3 efficiently suppressed the EMT process and rearranged the cytoskeletal proteins in HNSCC cells.

Inhibition of VEGFR2 attenuates the migration, invasion and EMT of HNSCC cells in vitro. Previous studies have demonstrated that constitutively activated STAT3 plays a pivotal role in tumor angiogenesis through upregulating VEGF expression $(30,31)$. Recent reports showed that inhibition of STAT3 
activity could downregulate VEGF and VEGFR2 expression (32), while VEGFR2 mediates cancer metastasis (33). We therefore hypothesized that VEGFR2 may act as a downstream factor of STAT3 and mediate the EMT process in HNSCC. We transfected UM1 and CAL27 cells with siKDR. As presented in Fig. 4E, the expression of VEGFR2 was notably lower after siKDR transfection in UM1 and CAL27 cells. Similarly, VEGF expression was also reduced. Intriguingly, the Transwell assay showed that siKDR treatment significantly inhibited UM1 and CAL27 cells migration and invasion compared with the control $(\mathrm{P}<0.001$; Fig. 4B). The results of the scratch-wound assays showed a significant reduction in the migration in both UM1 and CAL27 cell lines compared with the control $(\mathrm{P}<0.001$ and $\mathrm{P}<0.01$; Fig. 4A). To further demonstrate that VEGFR2 mediates the invasion and migration of HNSCC cells, we knocked down VEGFR2 expression in UM1 and CAL27 cells with the EMT-phenotype. Western blotting showed that the EMT marker, E-cadherin, was notably upregulated, while the expression of N-cadherin, Vimentin and Twist-1 was markedly downregulated in the siKDR-treated group (Fig. 4E). Immunofluorescence staining demonstrated that the expression of E-cadherin was increased, and that of $\mathrm{N}$-cadherin and Vimentin was decreased (Fig. 4C). In addition, the levels of F-actin in siKDR-treated group were markedly lowered and caused the changes in the cellular morphology compared with the control group (Fig. 4D).

Inhibition of the EZH2/STAT3/VEGFR2 axis suppresses the growth of HNSCC xenograft tumors and EMT in vivo. To examine whether inhibition of EZH2/STAT3/VEGFR2 axis reverses EMT in vivo, we established HNSCC xenograft tumors in immunocompromised mice using the CAL27 cell line. Compared with DMSO-treated tumors, the DZNep-treated group exhibited significantly lower tumor volume and tumor weight compared with the control $(\mathrm{P}<0.05$; Fig. 5A-C). In addition, the volume and weight of tumors of the Stattic group [a small molecule inhibitor selectively inhibits the expression levels of p-STAT3 (Try705), without any effect on total STAT3], those of the Stattic + DZNep, and Apatinib groups (a highly potent tyrosine-kinase inhibitor targeting VEGFR2) were also significantly reduced compared with DMSO group $(\mathrm{P}<0.01, \mathrm{P}<0.01, \mathrm{P}<0.05$; Fig. 5A-C). Interestingly, $\mathrm{H} \& \mathrm{E}$ staining in DMSO-treated CAL27 tumors had crowded nuclei with hyperchromatism and pleomorphism, while in the DZNep-treated group the nucleus of tumor cells exhibited a reduced degree of atypia. Moreover, IHC staining showed decreased expression of EZH2, p-STAT3 (Tyr705), VEGF, VEGFR2, N-cadherin and Vimentin, and increased expression of E-cadherin, without notable alterations in total STAT3 levels in DZNep-treated CAL27 tumors (Fig. 5D and E). These results further validated that EZH2, STAT3 and VEGFR2 were closely linked and inhibition of this axis could profoundly impact the EMT of HNSCC cells.

\section{Discussion}

The development of novel targets for the treatment of metastatic HNSCC is necessary. Accumulating evidence suggests that EMT and cancer metastatic ability are inextricably associated during cancer development $(4,5)$; however, the molecular mechanisms underlying EMT in HNSCC remain unclear. In this study, we reported that an EZH2-centered axis regulates HNSCC progression, as manifested by our observations that targeting the EZH2/STAT3/VEGFR2 pathway suppresses HNSCC metastatic progression both in vitro and in vivo.

Tumor metastasis is the major cause of cancer-associated mortality and the process has been divided into at least four steps: Local invasion, intravasation, extravasation and metastatic colonization $(3,4)$. EMT is considered to play a critical role in initiating cancer metastasis, in association with loss of E-cadherin expression in HNSCC, particularly in patients with lymph node metastasis and with poor prognosis $(4,34)$. As of its role in epigenetic gene silencing and chromatin remodeling, EZH2 is crucial in tumorigenesis and cancer progression $(13,14)$. Accumulating evidence suggests that EZH2 is closely related to EMT in several cancer types (35), and our previous study demonstrated that EZH2 is upregulated in HNSCC and acts as a negative prognostic factor for patients with HNSCC (11). The present study, to the best of our knowledge, we are the first to demonstrate that inhibition of EZH2 reverses EMT in HNSCC cells and xenograft tumors by regulating the STAT3/VEGFR2 axis in vitro and in vivo.

Transcription factor STAT3 was shown to be abnormally activated in various cancer types. With a well-known role in promoting tumor cell proliferation, survival, tumor invasion, angiogenesis and immunosuppression, STAT3 also promotes cancer development through inflammation, stem cells, obesity, and the pre-metastatic niche formation (16-19,36-38). Our previous study demonstrated that targeting STAT3 by using HJC0152 and other inhibitors significantly suppressed the growth and invasion of HNSCC via regulating the microRNA-21/ $\beta$-catenin axis (28). Recent research has shown that EZH2 could enhance cancer cell invasion and metastasis through regulating the activation of STAT3 (39). One study reported that targeting EZH2/STAT3 signals with EZH2 inhibitor GSK-126 or ASC-J9 ${ }^{\circledR}$ (a novel androgen receptor degradation enhancer) decreases the invasion of prostate cancer stem/progenitor cells (40). Another study validated that phosphorylation of $\mathrm{EZH} 2$ at serine 21 via AKT promotes STAT3 methylation and enhances the activation of STAT3 (20). STAT3 is closely associated with VEGF $(24,25)$ or cyclin dependent kinase 2 (41) in cancer progression. Lee et al (42) demonstrated that arsenic herbal mixture PROS exerts antitumor effects by targeting STAT3/VEGF/CDK2 axis in non-small lung cancer cells.

In our in vivo experiments, we found that inhibition of EZH2 in HNSCC cells resulted in the suppression of p-STAT3 (Tyr705) expression, along with reduced expression of VEGF, VEGFR2, and EMT-related markers but E-cadherin was elevated. In contrast, overexpression of EZH2 resulted in increased the expression p-STAT3 (Tyr705), VEGF, and VEGFR2. Additionally, after targeting STAT3 by siRNA, the expression of p-STAT3 (Tyr705), VEGF, VEGFR2, and EMT-related markers except E-cadherin were similarly attenuated. Consistently, EZH2 inhibition decreased the migration and invasion of HNSCC cells, similar results were found in the HNSCC cells treated with siSTAT3. Angiogenesis plays an important role in tumor growth and metastasis (24). VEGF and its receptors, mainly VEGFR2, have a crucial role in promoting angiogenesis (43). Niu et al (30) reported that constitutively activated STAT3 upregulates VEGF 
A

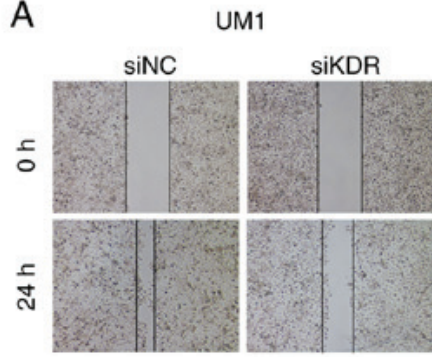

B

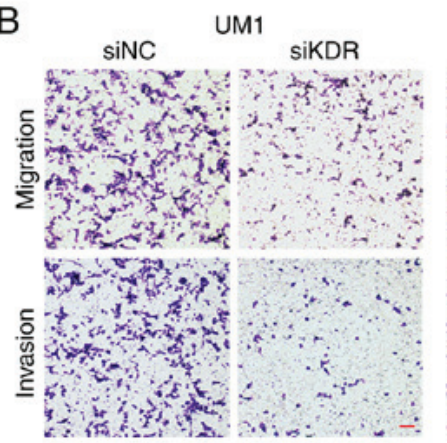

CAL27

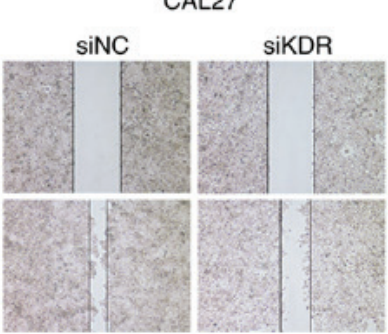

siNC

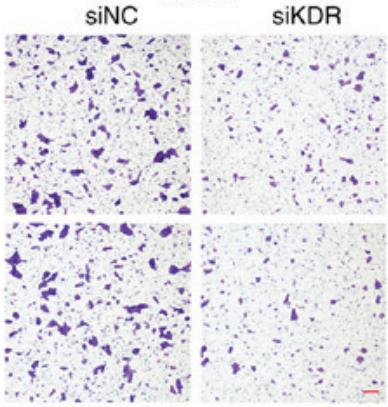

UM1 口0 h
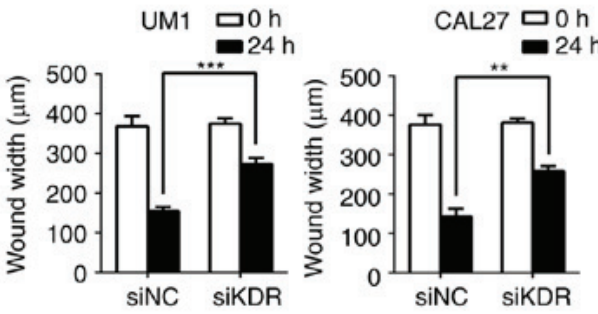

C
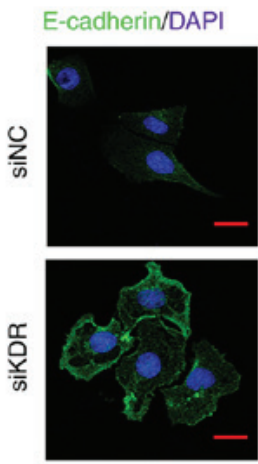

UM1

$\mathrm{N}$-cadherin/DAPI
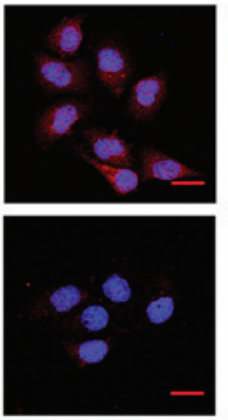

Vimentin/DAPI
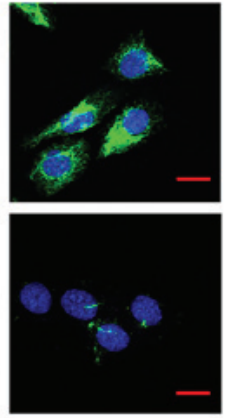

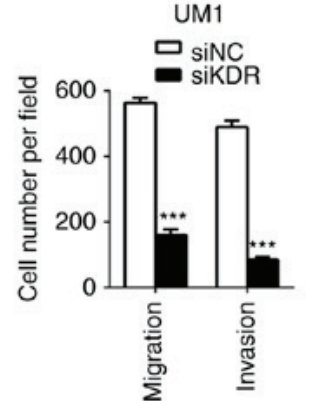

CAL27

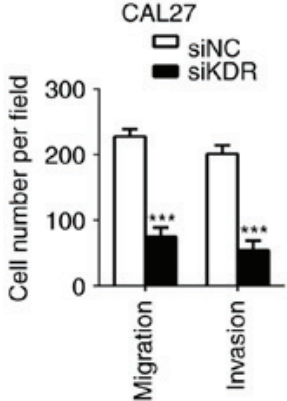

E-cadherin/DAPI

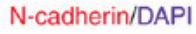

Vimentin/DAPI
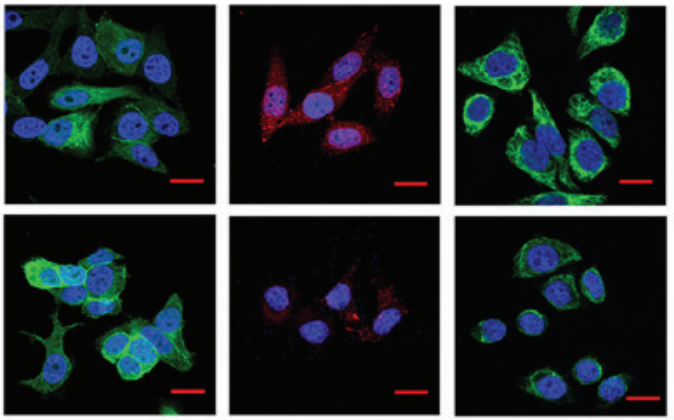

D
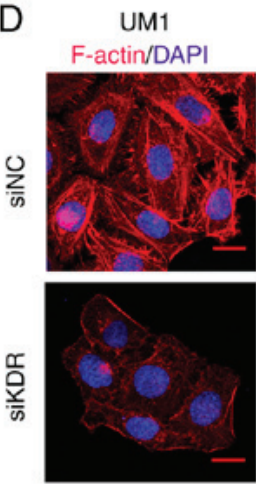

CAL27
F-actin/DAPI
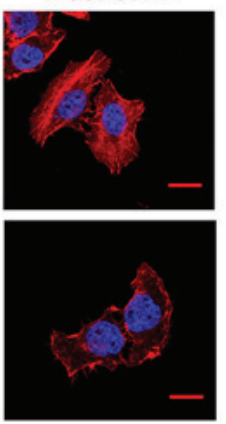

E

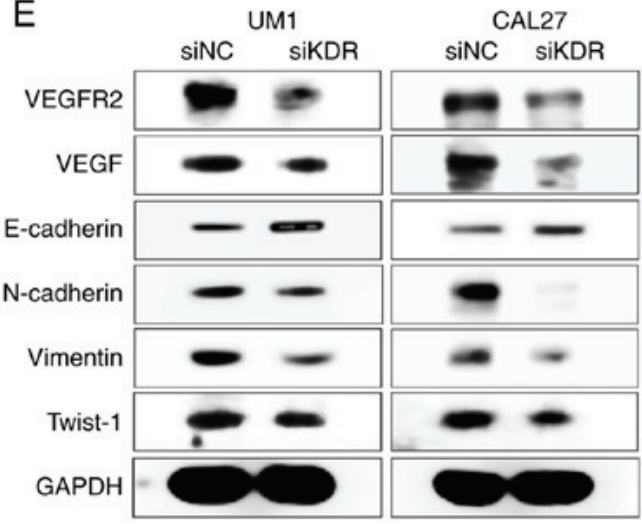

Figure 4. VEGFR2 regulates EMT in HNSCC cells in vitro. (A) Wound-healing assays performed to investigate the migration in siKDR-treated UM1 and CAL27 cells $\left({ }^{* *} \mathrm{P}<0.01,{ }^{* * *} \mathrm{P}<0.001\right)$. (B) Transwell assays performed to show migration (without Matrigel) and invasion (with Matrigel) in the siKDR-treated group. ${ }^{* * *} \mathrm{P}<0.001$ vs. siNC. Scale bar, $50 \mu \mathrm{m}$. (C) Confocal immunofluorescence analysis performed to show the change in the expression of EMT-related markers in siKDR-treated cells compared with the scramble cells. Scale bar, $20 \mu \mathrm{m}$. (D) Confocal immunofluorescence analysis to show F-actin rearrangement in siKDR-treated HNSCC cells. Scale bar, $20 \mu \mathrm{m}$. (E) Western blot analysis was performed to show the effects of VEGFR2 knockdown on the expression of E-cadherin, VEGF, N-cadherin, Vimentin and Twist-1 in UM1 and CAL27 cell lines. EMT, epithelial-mesenchymal transition; HNSCC, head and neck squamous cell carcinoma; NC, negative control; si, small interfering RNA; Twist-1, Twist-related protein 1; VEGF, vascular endothelial growth factor; VEGFR2, VEGF receptor 2; KDR, kinase insert domain receptor.

expression and thereby induces tumor angiogenesis. Xue et al (32) demonstrated that STAT3 inhibition decreases the expression of VEGF and VEGFR2. In current study, we found that targeting STAT3 by siRNA in HNSCC cells attenuates the expression of VEGF and VEGFR2, suggesting that the VEGF/VEGFR2 axis served as the downstream effectors of STAT3 in HNSCC cells. Finally, we demonstrated that knocking-down of VEGFR2 by siKDR decreased the expression of EMT-related markers 

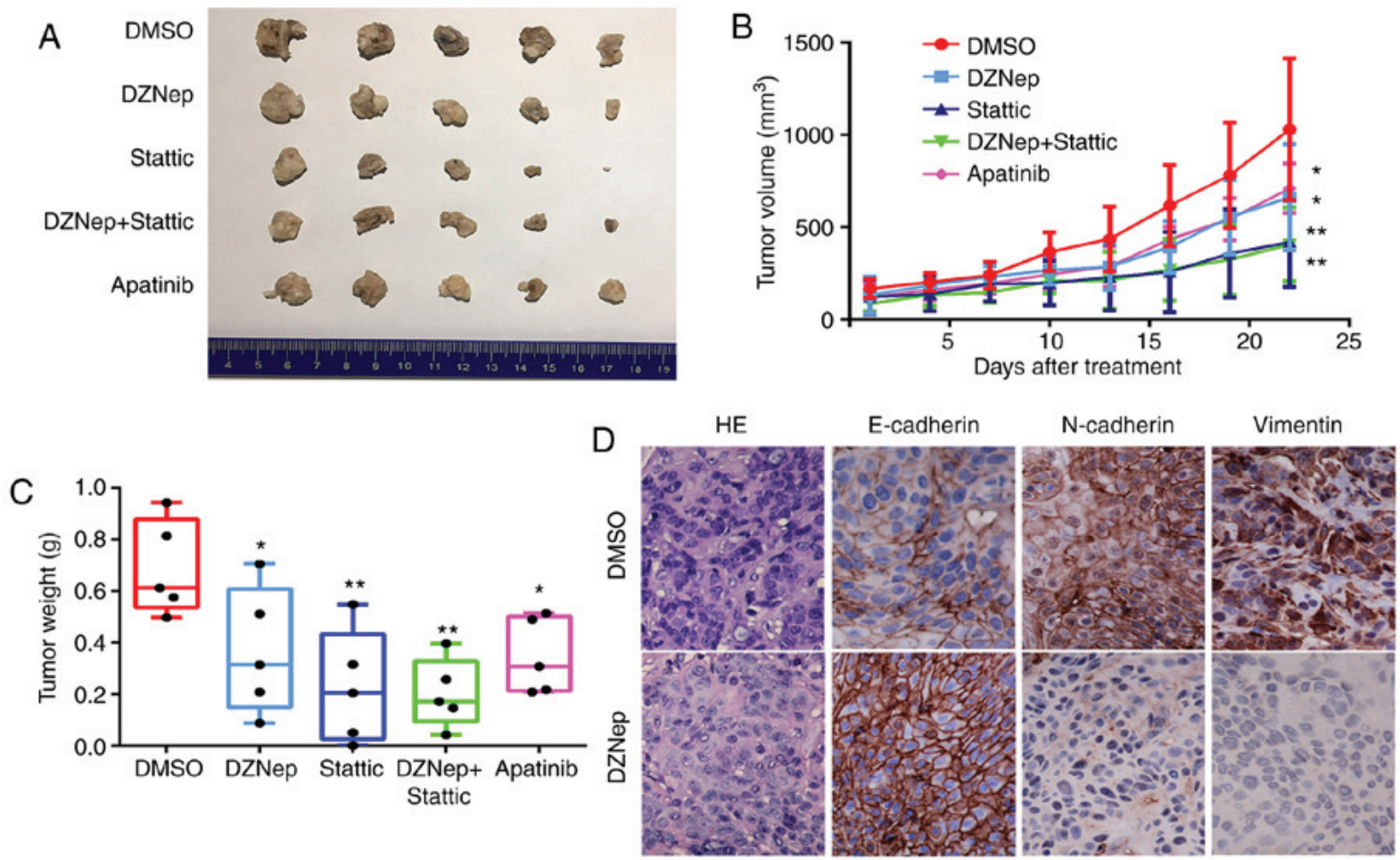

E
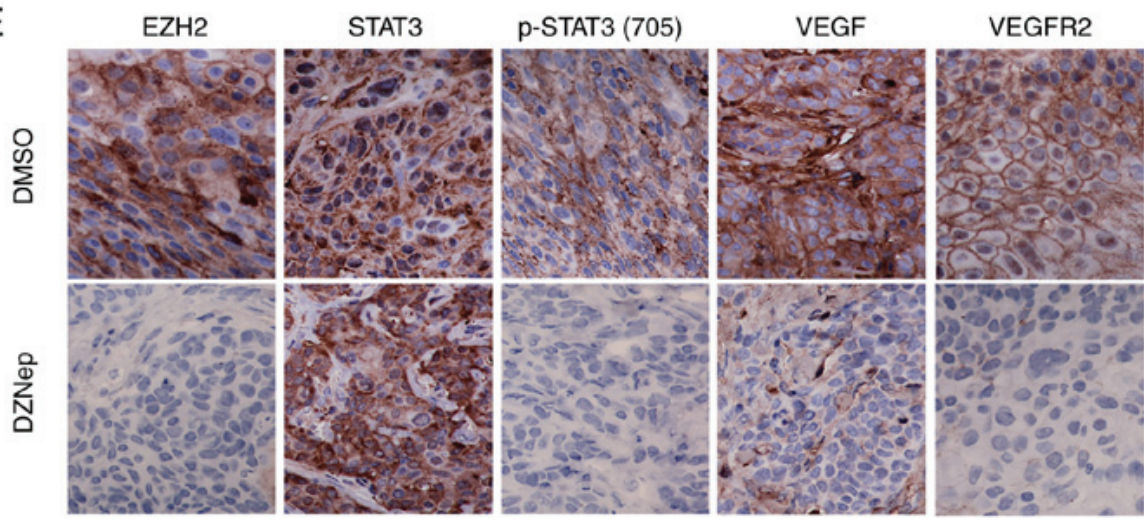

Figure 5. Targeting the EZH2/STAT3/VEGFR2 axis inhibits the growth of HNSCC xenograft tumors and reverses EMT in HNSCC tumors in vivo. (A-C) The volume and weight of CAL27 xenograft tumors treated with DMSO, DZNep, Stattic, DZNep + Stattic, and Apatinib (two-way ANOVA; " $\mathrm{P}<0.05$, ${ }^{* * *} \mathrm{P}<0.01$ vs. DMSO). (D) Immunohistochemical staining was performed to show EMT-related markers N-cadherin, Vimentin, and E-cadherin expression in the DZNep-treated group (magnification, x400). (E) Immunohistochemical staining performed to determine the expression of EZH2, STAT3, p-STAT3 (Tyr705), VEGF, and VEGFR2 in the DZNep-treated group (magnification, 400). DMSO, dimethyl sulfoxide; DZNep, 3-deazaneplanocin A; EMT, epithelial-mesenchymal transition; EZH2, enhancer of zeste homolog 2; HNSCC, head and neck squamous cell carcinoma; p, phosphorylated; STAT3, signal transducer and activator of transcription factor 3; VEGF, vascular endothelial growth factor; VEGFR2, VEGF receptor 2.

but increased E-cadherin, meanwhile the invasion and migration abilities of HNSCC cells were attenuated. These findings suggest that targeting EZH2 reverses EMT in HNSCC cells via regulating STAT3/VEGFR2 axis.

The results of our in vivo study consistently confirmed the in vitro findings. EZH2 inhibition suppressed the growth and EMT process of CAL27 cell-derived xenograft tumors. To the best of our knowledge, our findings indicated that the EZH2/STAT3/VEGFR2 axis plays a critical role in promoting the metastasis of HNSCC. One potential application of this study is the targeting of the EZH2/STAT3/VEGFR2 axis as a novel and effective approach in treating invasive, progressive, and metastatic HNSCC.

In summary, we demonstrated that $\mathrm{EZH} 2$ promotes the progression and EMT process of HNSCC via regulating
STAT3 and VEGFR2. This EZH2-centered mechanism in HNSCC metastasis may serve as a rationale to develop general and/or specific inhibitory approaches for targeting the EZH2/STAT3/VEGFR2 axis effectively control HNSCC progression and metastasis.

\section{Acknowledgements}

Not applicable.

\section{Funding}

This work was supported by grants from the National Natural Science Foundation of China (grant nos. 81572492, 81872206, 81872495, 81702678 and 81673026), the National Clinical 
Research Center for Cancer (NCRCC) and Supported by Special Program of Talents Development for Excellent Youth Scholars in Tianjin.

\section{Availability of data and materials}

All data generated or analyzed during this study are included in this published article.

\section{Authors' contributions}

$\mathrm{MZ}, \mathrm{XH}, \mathrm{YX}, \mathrm{CW}, \mathrm{JC}$ and LK performed the experiments and data analysis, and wrote the manuscript. LK, SS, YR and RJ were involved in revising the manuscript critically for important intellectual content. XZ and LZ also gave final approval of the version to be published. All authors read and approved the final manuscript.

\section{Ethics approval and consent to participate}

The present study was approved by the Institutional Animal Care and Use Committee of Tianjin Medical University Cancer Institute and Hospital (Tianjin, China).

\section{Patient consent for publication}

Not applicable.

\section{Competing interests}

The authors have declared that no competing interest exists.

\section{References}

1. Ferlay J, Shin HR, Bray F, Forman D, Mathers C and Parkin DM: Estimates of worldwide burden of cancer in 2008: GLOBOCAN 2008. Int J Cancer 127: 2893-2917, 2010.

2. Leemans CR, Braakhuis BJ and Brakenhoff RH: The molecular biology of head and neck cancer. Nat Rev Cancer 11: 9-22, 2011

3. Thiery JP, Acloque H, Huang RY and Nieto MA: Epithelial-mesenchymal transitions in development and disease. Cell 139: 871-890, 2009.

4. Smith A, Teknos TN and Pan Q: Epithelial to mesenchymal transition in head and neck squamous cell carcinoma. Oral Oncol 49: 287-292, 2013.

5. Sun SS, Zhou X, Huang YY, Kong LP, Mei M, Guo WY, Zhao MH, Ren Y, Shen Q and Zhang L: Targeting STAT3/miR-21 axis inhibits epithelial-mesenchymal transition via regulating CDK5 in head and neck squamous cell carcinoma. Mol Cancer 14: 213, 2015.

6. Cao R, Wang L, Wang H, Xia L, Erdjument-Bromage H, Tempst P, Jones RS and Zhang Y: Role of histone $\mathrm{H} 3$ lysine 27 methylation in polycomb-group silencing. Science 298: 1039-1043, 2002.

7. Kuzmichev A, Nishioka K, Erdjument-Bromage H, Tempst $P$ and Reinberg D: Histone methyltransferase activity associated with a human multiprotein complex containing the enhancer of zeste protein. Genes Dev 16: 2893-2905, 2002.

8. Karanikolas BD, Figueiredo ML and Wu L: Comprehensive evaluation of the role of EZH2 in the growth, invasion, and aggression of a panel of prostate cancer cell lines. Prostate 70 675-688, 2010.

9. Ahani N, Shirkoohi R, Rokouei M, Alipour Eskandani M and Nikravesh A: Overexpression of enhancer of zeste human homolog 2 (EZH2) gene in human cytomegalovirus positive glioblastoma multiforme tissues. Med Oncol 31: 252, 2014.

10. Reijm EA, Timmermans AM, Look MP, Meijer-van Gelder ME, Stobbe CK, van Deurzen CH, Martens JW, Sleijfer S, Foekens JA, Berns PM and Jansen MP: High protein expression of EZH2 is related to unfavorable outcome to tamoxifen in metastatic breast cancer. Ann Oncol 25: 2185-2190, 2014.
11. Zhou X, Ren Y, Kong LP, Cai GH, Sun SS, Song WZ, Wang Y, Jin R, Qi L, Mei M, et al: Targeting EZH2 regulates tumor growth and apoptosis through modulating mitochondria dependent cell-death pathway in HNSCC. Oncotarget 6: 33720-33732, 2015.

12. Sun J, Zheng G, Gu Z and Guo Z: MiR-137 inhibits proliferation and angiogenesis of human glioblastoma cells by targeting EZH2. J Neurooncol 122: 481-489, 2015.

13. Luo H, Jiang Y, Ma S, Chang H, Yi C, Cao H, Gao H, Guo H, Hou J, Yan J, et al: EZH2 promotes invasion and metastasis of laryngeal squamous cells carcinoma via epithelial-mesenchymal transition through H3K27me3. Biochem Biophys Res Commun 479: 253-259, 2016.

14. Mu Z, Li H, Fernandez SV, Alpaugh KR, Zhang R and Cristofanilli M: EZH2 knockdown suppresses the growth and invasion of human inflammatory breast cancer cells. J Exp Clin Cancer Res 32: 70, 2013.

15. Wang C, Liu X, Chen Z, Huang H, Jin Y, Kolokythas A, Wang A, Dai Y, Wong DT and Zhou X: Polycomb group protein EZH2-mediated E-cadherin repression promotes metastasis of oral tongue squamous cell carcinoma. Mol Carcinog 52: 229-236, 2013.

16. Frank DA: STAT3 as a central mediator of neoplastic cellular transformation. Cancer Lett 251: 199-210, 2007.

17. Bar-Natan M, Nelson EA, Xiang M and Frank DA: STAT signaling in the pathogenesis and treatment of myeloid malignancies. JAKSTAT 1: 55-64, 2012.

18. Xiong A, Yang Z, Shen Y, Zhou J and Shen Q: Transcription factor STAT3 as a novel molecular target for cancer prevention. Cancers (Basel) 6: 926-957, 2014.

19. Masuda M, Suzui M, Yasumatu R, Nakashima T, Kuratomi Y, Azuma K, Tomita K, Komiyama S and Weinstein IB: Constitutive activation of signal transducers and activators of transcription 3 correlates with cyclin D1 overexpression and may provide a novel prognostic marker in head and neck squamous cell carcinoma. Cancer Res 62: 3351-3355, 2002.

20. Kim E, Kim M, Woo DH, Shin Y, Shin J, Chang N, Oh YT, Kim H, Rheey J, Nakano I, et al: Phosphorylation of EZH2 activates STAT3 signaling via STAT3 methylation and promotes tumorigenicity of glioblastoma stem-like cells. Cancer Cell 23: 839-852, 2013.

21. Dasgupta M, Dermawan JK, Willard B and Stark GR: STAT3-driven transcription depends upon the dimethylation of K49 by EZH2. Proc Natl Acad Sci USA 112: 3985-3990, 2015.

22. Neuchrist C, Erovic BM, Handisurya A, Steiner GE, Rockwell P, Gedlicka C and Burian M: Vascular endothelial growth factor receptor 2 (VEGFR2) expression in squamous cell carcinomas of the head and neck. Laryngoscope 111: 1834-1841, 2001.

23. Lu W, Chen H, Ye F, Wang F and Xie X: VEGF induces phosphorylation of STAT3 through binding VEGFR2 in ovarian carcinoma cells in vitro. Eur J Gynaecol Oncol 27: 363-369, 2006.

24. Zhao M, Gao FH, Wang JY, Liu F, Yuan HH, Zhang WY and Jiang B: JAK2/STAT3 signaling pathway activation mediates tumor angiogenesis by upregulation of VEGF and bFGF in non-small-cell lung cancer. Lung Cancer 73: 366-374, 2011.

25. Xie TX, Huang FJ, Aldape KD, Kang SH, Liu M, Gershenwald JE, Xie K, Sawaya R and Huang S: Activation of stat3 in human melanoma promotes brain metastasis. Cancer Res 66: 3188-3196, 2006.

26. U.S. National Institutes of Health: Laboratory animal welfare: Public Health Service policy on humane care and use of laboratory animals by awardee institutions; notice. Fed Regist 50: 19584-19585, 1985.

27. Feldman AT and Wolfe D: Tissue processing and hematoxylin and eosin staining. Methods Mol Biol 1180: 31-43, 2014.

28. Wang Y, Wang S, Wu Y, Ren Y, Li Z, Yao X, Zhang C, Ye N, Jing C, Dong J, et al: Suppression of the growth and invasion of human head and neck squamous cell carcinomas via regulating STAT3 signaling and the miR-21/ $\beta$-catenin axis with HJC0152. Mol Cancer Ther 16: 578-590, 2017.

29. Nieto MA: The ins and outs of the epithelial to mesenchymal transition in health and disease. Annu Rev Cell Dev Biol 27: 347-376, 2011.

30. Niu G, Wright KL, Huang M, Song L, Haura E, Turkson J, Zhang S, Wang T, Sinibaldi D, Coppola D, et al: Constitutive Stat3 activity up-regulates VEGF expression and tumor angiogenesis. Oncogene 21: 2000-2008, 2002. 
31. Wei D, Le X, Zheng L, Wang L, Frey JA, Gao AC, Peng Z, Huang S, Xiong HQ, Abbruzzese JL and Xie K: Stat 3 activation regulates the expression of vascular endothelial growth factor and human pancreatic cancer angiogenesis and metastasis. Oncogene 22: 319-329, 2003.

32. Xue C, Xie J, Zhao D, Lin S, Zhou T, Shi S, Shao X, Lin Y, Zhu B and Cai X: The JAK/STAT3 signalling pathway regulated angiogenesis in an endothelial cell/adipose-derived stromal cell co-culture, 3D gel model. Cell Prolif 50: 2017.

33. Balakrishnan S, Bhat FA, Raja Singh P, Mukherjee S, Elumalai P Das S, Patra CR and Arunakaran J: Gold nanoparticle-conjugated quercetin inhibits epithelial-mesenchymal transition, angiogenesis and invasiveness via EGFR/VEGFR-2-mediated pathway in breast cancer. Cell Prolif 49: 678-697, 2016.

34. Diniz-Freitas M, Garcia-Caballero T, Antúnez-López J, Gándara-Rey JM and Garcia-Garcia A: Reduced E-cadherin expression is an indicator of unfavourable prognosis in oral squamous cell carcinoma. Oral Oncol 42: 190-200, 2006.

35. Chang JW, Gwak SY, Shim GA, Liu L, Lim YC, Kim JM, Jung MG and Koo BS: EZH2 is associated with poor prognosis in head-and-neck squamous cell carcinoma via regulating the epithelial-to-mesenchymal transition and chemosensitivity. Oral Oncol 52: 66-74, 2016.

36. Priceman SJ, Kujawski M, Shen SD, Cherryholmes GA, Lee H, Zhang C, Kruper L, Mortimer J, Jove R, Riggs AD and Yu H: Regulation of adipose tissue $\mathrm{T}$ cell subsets by Stat 3 is crucial for diet-induced obesity and insulin resistance. Proc Natl Acad Sci USA 110: 13079-13084, 2013.
37. Schroeder A, Herrmann A, Cherryholmes G, Kowolik C, Buettner R, Pal S, Yu H, Müller-Newen G and Jove R: Loss of androgen receptor expression promotes a stem-like cell phenotype in prostate cancer through STAT3 signaling. Cancer Res 74: 1227-1237, 2014.

38. Yu H, Lee H, Herrmann A, Buettner R and Jove R: Revisiting STAT3 signalling in cancer: New and unexpected biological functions. Nat Rev Cancer 14: 736-746, 2014

39. Xu Z, Sun Y, Guo Y, Qin G, Mu S, Fan R, Wang B, Gao W, Wu H, Wang $G$ and Zhang Z: NF-YA promotes invasion and angiogenesis by upregulating EZH2-STAT3 signaling in human melanoma cells. Oncol Rep 35: 3630-3638, 2016.

40. Wen S, Tian J, Niu Y, Li L, Yeh S and Chang C: ASC-J9(®), and not casodex or enzalutamide, suppresses prostate cancer stem/progenitor cell invasion via altering the EZH2-STAT3 signals. Cancer Lett 376: 377-386, 2016.

41. Steinman RA, Wentzel A, Lu Y, Stehle C and Grandis JR: Activation of Stat 3 by cell confluence reveals negative regulation of Stat 3 by cdk2. Oncogene 22: 3608-3615, 2003.

42. Lee H, Lee HJ, Bae IJ, Kim JJ and Kim SH: Inhibition of STAT3/VEGF/CDK2 axis signaling is critically involved in the antiangiogenic and apoptotic effects of arsenic herbal mixture PROS in non-small lung cancer cells. Oncotarget 8: 101771-101783, 2017

43. Hoeben A, Landuyt B, Highley MS, Wildiers H, Van Oosterom AT and De Bruijn EA: Vascular endothelial growth factor and angiogenesis. Pharmacol Rev 56: 549-580, 2004. 